\title{
Hospital-acquired influenza in an Australian sentinel surveillance system
}

\section{Nenad Macesic MB BS, BA Infectious Diseases Registrar \\ Tom C Kotsimbos \\ MB BS, FRACP, MD, Respiratory and Transplant Physician $^{2}$ \\ Paul Kelly MB BS, PhD, FAFPHM Chief Medical Officer \\ Allen C Cheng MB BS, FRACP, PhD, Infectious Disease Physician $^{4}$ \\ On behalf of the FluCAN investigators* \\ 1 Infectious Diseases Unit, Alfred Health Melbourne, VIC. \\ 2 Allergy, Immunology and Respiratory Medicine Unit, Alfred Health, Melbourne, VIC. \\ 3 ACT Health Directorate Canberra, ACT. \\ 4 Department of Epidemiology and \\ Preventive Medicine \\ Monash University, Melbourne, VIC \\ allen.cheng@ monash.edu \\ *Allen Cheng, Mark Holmes Tom Kotsimbos, Louis Irving Simon Brown, Grant Waterer, Tony Korman, Deb Friedman, \\ Sanjaya Senanayake Paul Kelly.}

MJA 2013; 198: 370-372 doi: 10.5694/mjal2.11687

Editorial p 349 Research p 373 osocomial influenza has been associated with significant morbidity, mortality and cost due to increased length of stay. It is likely to be under-recognised because of rapid turnover of patients and delays in diagnosis. Most previous reports of nosocomial influenza have involved known case clusters. ${ }^{1}$ We aimed to review cases of nosocomial influenza detected in a hospital-based surveillance program.

\section{Methods}

The Influenza Complications Alert Network (FluCAN) is a sentinel surveillance system that prospectively collects data on adult patients hospitalised with confirmed influenza. ${ }^{2}$ In 2010, this system involved 15 hospitals in all Australian jurisdictions and, in 2011, eight hospitals in Victoria, the Australian Capital Territory, South Australia and Western Australia. Surveillance was performed between April and November of each year.

Influenza was diagnosed using nucleic-acid detection from respiratory samples. Testing was performed at the discretion of treating clinicians but was encouraged for influenza-like illnesses because of infection prevention considerations. Seasonal influenza subtypes were not recorded, but other surveillance systems suggest that pandemic (H1N1) 2009 influenza was the major circulating strain in 2010 and 2011. ${ }^{3}$ Patients were identified by surveillance of laboratory testing logs. A nosocomial case was defined as polymerase chain reactionconfirmed influenza where the onset of symptoms was more than 2 days after the patient's admission or, if this was not known, where the date of the positive test was more than 7 days after admission.

Continuous measures were compared using the Mann-Whitney $U$

\section{Abstract}

Objective: To review cases of nosocomial influenza and compare the epidemiology, clinical characteristics and outcomes with community-acquired cases.

Design, setting and participants: Prospective case series of adults hospitalised with influenza during April - November of 2010 and 2011 using a hospital-based sentinel surveillance system. A nosocomial case was defined as polymerase chain reaction-confirmed influenza where symptom onset was more than 2 days after admission or, if this was not known, where the date of the positive test was more than 7 days after admission.

Main outcome measures: Demographic, clinical and outcome measures for patients with nosocomial influenza compared with patients admitted with community-acquired influenza.

Results: In 2010-2011, 598 cases of influenza were detected, of which 26 $(4.3 \%)$ were nosocomial. All patients with nosocomial influenza had chronic comorbidities, compared with $71.7 \%$ of patients (410/572) with communityacquired influenza $(P=0.001)$. Similar proportions of community-acquired $(32.5 \%)$ and nosocomial (36.4\%) cases occurred in patients vaccinated in the current season. Clinical findings at time of enrolment did not differ between the two groups, with similar rates of fever, cough, chest pain and dyspnoea.

Compared with community-acquired cases, a higher proportion of patients with nosocomial influenza received neuraminidase inhibitors within 2 days of symptom onset (38.5\% v 15.9\%; $P=0.003$ ). Admission to intensive care took place in $21.3 \%$ and $23.1 \%$ of community-acquired and nosocomial cases, respectively. One death from nosocomial influenza occurred in a patient with end-stage respiratory disease.

Conclusions: Nosocomial influenza is uncommon but may be associated with severe disease. It may be partially preventable as patients frequently have comorbidities for which influenza vaccination is recommended. Patients, particularly those at high risk of complications, and their contacts (including health care workers) should be vaccinated to prevent severe disease.

test, and categorical variables with the $\chi^{2}$ test or Fisher exact test as appropriate. We calculated crude (unadjusted) odds ratios (ORs) for characteristics of patients with nosocomial and community-acquired influenza. All statistical analysis was performed using Stata 12.1 (StataCorp). Ethics approval for the study was obtained from all sites and the Australian National University.

\section{Results}

\section{Diagnosis of influenza}

Of 598 patients diagnosed with confirmed influenza in the 2010 and 2011 surveillance periods, 26 (4.3\%) had nosocomial influenza (Box 1). Cases were seen at multiple hospitals and were coincident with the presentation of community-acquired cases (data not shown). Compared with community-acquired cases, nosocomial cases were diagnosed sooner after onset of symptoms (Box 2). Symptom onset in the nosocomial group occurred a median of 12.5 days after admission.

\section{Demographic characteristics and comorbidities}

All patients with nosocomial influenza had chronic comorbidities, compared with $71.7 \%$ of communityacquired cases $(P=0.001)$ (Box 1). Patients with nosocomial influenza were significantly more likely to be immunosuppressed or have an underlying malignancy. Of the 22 
nosocomial cases where vaccination status was known, eight patients (36.4\%) had been vaccinated against influenza in the current season.

\section{Clinical characteristics and initial treatment}

Clinical findings at time of enrolment did not differ between the two groups, with similar rates of fever, cough, chest pain and dyspnoea (data not shown). Similar proportions of patients in both groups were treated with neuraminidase inhibitors. Treatment was initiated earlier in the nosocomial group, and a significantly higher proportion of this group received treatment within 2 days of onset of symptoms (Box 2).

\section{Course of illness}

The hospital stay included an admission to the intensive care unit (ICU) for $21.3 \%$ and $23.1 \%$ of patients with community-acquired and nosocomial influenza, respectively (Box 2). Of the six patients with nosocomial influenza who were cared for in the ICU, three were diagnosed before admission to the ICU.

\section{Mortality}

One patient, a man aged in his 40s with end-stage respiratory disease and renal disease requiring dialysis, died from nosocomial influenza. He had not been vaccinated against influenza. Influenza A (H3N2) was diagnosed on Day 18 of his admission, and he was treated with a 5-day course of oseltamivir. The patient died 2 weeks later from worsening respiratory failure.

\section{Discussion}

We found that nosocomial influenza was uncommon but may be severe. Our finding that $4.3 \%$ of hospitalised influenza patients had nosocomial influenza is similar to that from an observational cohort study in the United Kingdom in 2009-2010, which detected 30 nosocomial cases $(2.0 \%)$ in 1520 hospitalised patients with influenza at 75 hospitals. ${ }^{4}$ The low numbers and high severity of illness may suggest underdiagnosis of mild cases, patients presenting after discharge, or the susceptibility of hospitalised patients with significant

1 Demographic characteristics and comorbidities of patients with community-acquired or nosocomial influenza

\begin{tabular}{|c|c|c|c|}
\hline Variable & $\begin{array}{l}\text { Community-acquired } \\
\text { influenza }\end{array}$ & $\begin{array}{l}\text { Nosocomial } \\
\text { influenza }\end{array}$ & $\begin{array}{l}\text { Odds ratio } \\
(95 \% \mathrm{Cl})\end{array}$ \\
\hline Number of patients & 572 & 26 & \\
\hline Male & $273(47.7 \%)$ & $14(53.8 \%)$ & $1.3(0.5-3.1)$ \\
\hline Median age (range) & $44(0-93)$ & $52(26-89)$ & -* \\
\hline Indigenous & $24(4.2 \%)$ & $1(3.8 \%)$ & $0.9(0.0-6.1)$ \\
\hline Nursing home resident & $11(1.9 \%)$ & 0 & nd $(0-7.7)$ \\
\hline Pregnant & $32(5.6 \%)$ & $1(3.8 \%)$ & $0.7(0.0-4.4)$ \\
\hline Smoker & $120(21.0 \%)$ & $6(23.1 \%)$ & $1.1(0.4-3.0)$ \\
\hline Any chronic comorbidity & $410(71.7 \%)$ & $26(100.0 \%)$ & nd $(2.7-n d)$ \\
\hline Chronic obstructive pulmonary disease & $17(3.0 \%)$ & $1(3.8 \%)$ & $1.3(0.0-9.0)$ \\
\hline Asthma & $63(11.0 \%)$ & $2(7.7 \%)$ & $0.7(0.1-2.8)$ \\
\hline Diabetes & $84(14.7 \%)$ & $6(23.1 \%)$ & $1.7(0.6-4.7)$ \\
\hline Immunosuppression & $114(19.9 \%)$ & $13(50.0 \%)$ & $4.0(1.7-9.7)$ \\
\hline Malignancy & $33(5.8 \%)$ & $4(15.4 \%)$ & $3.0(0.7-9.5)$ \\
\hline Cardiac disease & $77(13.5 \%)$ & $2(7.7 \%)$ & $0.5(0.1-2.2)$ \\
\hline Neurological disease & $51(8.9 \%)$ & $1(3.8 \%)$ & $0.4(0.0-2.6)$ \\
\hline Renal disease & $43(7.5 \%)$ & $4(15.4 \%)$ & $2.2(0.5-7.0)$ \\
\hline Vaccinated in current season & $101 / 311(32.5 \%)$ & $8 / 22(36.4 \%)$ & $1.2(0.4-3.1)$ \\
\hline
\end{tabular}

\section{Diagnosis and treatment of community-acquired or nosocomial influenza}

\begin{tabular}{|c|c|c|c|c|}
\hline Variable & $\begin{array}{l}\text { Community-acquired } \\
\text { influenza* }\end{array}$ & $\begin{array}{l}\text { Nosocomial } \\
\text { influenza }^{\dagger}\end{array}$ & $\begin{array}{l}\text { Odds ratio } \\
(95 \% \mathrm{Cl})\end{array}$ & $P$ \\
\hline $\begin{array}{l}\text { Median days to diagnosis from onset of } \\
\text { symptoms (range) }\end{array}$ & $4(0-41)$ & $1.5(0-8)$ & & $<0.001$ \\
\hline Delay to testing $\leqslant 2$ days & $173(30.2 \%)$ & $17(65.4 \%)$ & $4.4(1.8-11.3)$ & $<0.001$ \\
\hline $\begin{array}{l}\text { Median days hospitalised before onset of } \\
\text { influenza symptoms (range) }\end{array}$ & na & $12.5(3-64)$ & & - \\
\hline \multicolumn{5}{|l|}{ Influenza type } \\
\hline Influenza A & $514(89.9 \%)$ & $24(92.3 \%)$ & $1.4(0.3-12.1)$ & 0.51 \\
\hline Influenza B & $58(10.1 \%)$ & $2(7.7 \%)$ & $0.7(0.1-3.1)$ & 0.69 \\
\hline Treated with oseltamivir or zanamivir & $337 / 529(63.7 \%)$ & $16 / 25(64.0 \%)$ & $1.0(0.4-2.7)$ & 0.93 \\
\hline $\begin{array}{l}\text { Median days to treatment from symptom } \\
\text { onset (IQR) }\end{array}$ & $4(2-6)$ & $2(2-3)$ & & 0.004 \\
\hline Delay to treatment $\leqslant 2$ days & $91(15.9 \%)$ & $10(38.5 \%)$ & $3.3(1.3-8.0)$ & 0.003 \\
\hline Treated with antibiotics & 249/271 (91.9\%) & $7 / 11(63.6 \%)$ & $0.2(0.0-0.8)$ & 0.002 \\
\hline Admitted to intensive care unit & $122(21.3 \%)$ & $6(23.1 \%)$ & $1.1(0.4-2.9)$ & 0.91 \\
\hline Mechanical ventilation & $61 / 122(50.0 \%)$ & $4 / 6(66.7 \%)$ & $2.0(0.3-22.8)$ & 0.52 \\
\hline Vasopressor support & $65 / 122(53.3 \%)$ & $0 / 6$ & & 0.06 \\
\hline Died & $17(3.0 \%)$ & $1(3.8 \%)$ & $1.3(0.0-9.0)$ & 0.56 \\
\hline $\begin{array}{l}\text { Median days hospitalised before diagnosis } \\
\text { (IQR) }\end{array}$ & na & $15.5(7-32)$ & & - \\
\hline Median days hospitalised after diagnosis (IQR) & $4.0(2-7)$ & $27.5(3-35)$ & & $<0.001$ \\
\hline
\end{tabular}

comorbidities, particularly malignancy and immunosuppression, reflected in the nosocomial cases reported here. ${ }^{2,5}$

Poor outcomes have been described for nosocomial influenza, particularly in neonates and in patients who are immunosuppressed. ${ }^{4}$ In our study, the proportion of patients requiring ICU admission was similar in the nosocomial and community-acquired influenza groups. This differs from the UK cohort, ${ }^{4}$ where more than half the patients with nosocomial influenza required intensive care support. Mortality in nosocomial cases was also low in our study, with only one death noted, while mortality as high as $26.7 \%$ was reported in the UK study. ${ }^{4}$ 
Compared with community-acquired influenza cases, we noted a significantly longer length of stay for patients with nosocomial influenza, but there are likely numerous factors confounding this observation. Nosocomial influenza has previously been associated with an increased hospitalisation duration of 8 days, as well as increased use of diagnostics and treatment. ${ }^{5}$

Strategies to reduce nosocomial transmission of influenza include vaccination of patients before the winter season, vaccination of contacts including health care workers and visitors, more effective barrier precautions including improving hand hygiene compliance, and encouraging staff to stay away from work when unwell. Although influenza vaccination is publicly funded for older people and those with chronic comorbidities, protection is incomplete. We found that vaccination failed in a third of patients and twothirds were not vaccinated, consistent with studies estimating vaccine effectiveness against medically attended influenza $^{6}$ and hospitalisation with pandemic (H1N1) 2009 influenza at $49 \%-59 \% .^{7}$ Interventions to reduce transmission from infected contacts through barrier precautions and advice not to work or visit when unwell are hampered by infectivity before symptoms appear or by minimally symptomatic infection. Prophylactic antivirals are probably only feasible to contain outbreaks in closed facilities. Less than half of the patients with nosocomial influenza in our study received treatment with antivirals within 48 hours of symptom onset. This may reflect delays in diagnosis and the lack of efficacy of antivirals after 48 hours. This proportion is lower than in the UK cohort, where $57 \%$ of 21 patients received antivirals within 48 hours of onset of symptoms. $^{4}$

The proportion of health care workers known to be vaccinated in Australian hospitals has been reported at around $40 \% .^{8}$ Although two clusterrandomised trials found that mortality was lower in long-term care facilities where vaccination was actively promoted, the conclusions have been criticised because of methodological concerns. ${ }^{9}$ The results appeared to be internally inconsistent, with a large mortality benefit despite a lack of detection of significant influenza disease. There has been recent interest in making influenza vaccination a mandatory condition of working with patients, and this policy has been implemented with some success in the United States. ${ }^{10}$ It is not clear whether the patients identified in our study acquired infection from health care workers, from other infected patients or from visitors.

Our results suggest that a small proportion of influenza cases detected in hospitalised patients are acquired during the hospital stay, and that this may be associated with severe disease in a significant proportion of these patients. The overwhelming majority of nosocomial influenza occurs in patients with pre-existing medical illness that places them at high risk of complications.

Acknowledgements: We thank study staff at participating sites for their contribution.

Competing interests: No relevant disclosures

Received 21 Nov 2012, accepted 10 Mar 2013.

1 Chironna M, Tafuri S, Santoro N, et al. A nosocomial outbreak of 2009 pandemic influenza $A(\mathrm{HIN1})$ in a paediatric oncology ward in Italy, October-November 2009. Euro Surveill 2010; 15: pii 19454.

2 Kelly PM, Kotsimbos T, Reynolds A, et al. FluCAN 2009: initial results from sentinel surveillance for adult influenza and pneumonia in eight Australian hospitals. Med J Aust 2011; 194: 169-174.

3 Australian Government Department of Health and Ageing. Australian influenza surveillance report no. 10, 2012 - 29 September to 12 October 2012. http://www.health.gov.au/internet/main/ publishing.nsf/Content/cda-surveil-ozfluflucurr.htm (accessed Jan 2013).

4 Enstone JE, Myles PR, Openshaw PJM, et al. Nosocomial pandemic (HINI) 2009, United Kingdom, 2009-2010. Emerg Infect Dis 2011; 17: 592-598.

5 Serwint JR, Miller RM. Why diagnose influenza infections in hospitalized pediatric patients? Pediatr Infect Dis J 1993; 12: 200-204.

6 Osterholm MT, Kelley NS, Sommer A, et al. Efficacy and effectiveness of influenza vaccines: a systematic review and meta-analysis. Lancet Infect Dis 2012; 12: 36-44.

7 Cheng AC, Kotsimbos T, Kelly HA, et al. Effectiveness of HINI/O9 monovalent and trivalent influenza vaccines against hospitalization with laboratory-confirmed HINI/ 09 influenza in Australia: a test-negative case control study. Vaccine 2011; 29: 7320-7325.

8 Bull AL, Bennett N, Pitcher HC, et al. Influenza vaccine coverage among health care workers in Victorian public hospitals. Med J Aust 2007; 186: 185-186.

9 Thomas RE, Jefferson T, Lasserson TJ. Influenza vaccination for healthcare workers who work with the elderly. Cochrane Database Syst Rev 2010; (2): CD005187.

10 Rebmann T, Wright KS, Anthony J, et al. Seasonal influenza vaccine compliance among hospitalbased and nonhospital-based healthcare workers. Infect Control Hosp Epidemiol 2012; 33 : 243-249.

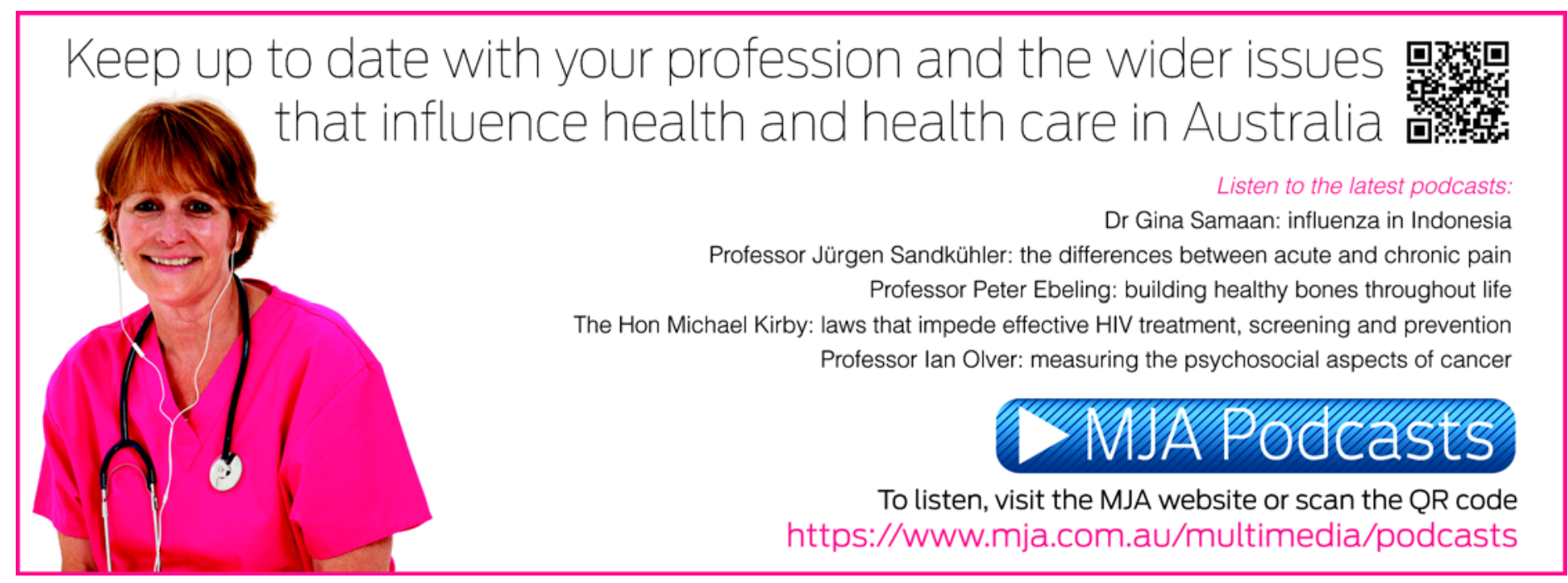

